\title{
Stochastic Synchronization in Self-Excited Systems
}

\author{
Katsutoshi Yoshida and Keijin Sato \\ Department of Mechanical Systems Engineering, Faculty of Engineering \\ Utsunomiya University, Tochigi 321-8585, JAPAN \\ Email:yoshidak@cc.utsunomiya-u.ac.jp
}

\begin{abstract}
This paper describes how to synchronize self-excited systems without coupling. The authors propose a method that enables us to produce a stable and precise synchronization of self-excited systems simply by adding noise. To achieve this, destruction of Hopf bifurcations of random invariant measures is investigated by means of a random van der Pol oscillator and a random nonlinear retarded ocsillator. The destruction results in the exact synchronization between the random self-excited systems. A quasi-periodic systems is also synchronized by the proposed method.
\end{abstract}

\section{Introduction}

Over the past few decades, numerous attempts have been made to synchronize dynamical systems. The main interest of this field seems to be in the question of how to couple dynamical systems to obtain desired types of synchronization. There are many approaches such as, deriving the phase diagram of coupled nonlinear oscillator [1], investigating noise enhancement $[2,3]$ and stochastic resonance [4] for synchronizations of coupled oscillators, and experimental studies using electric circuits [5,6] and laser systems [7].

In contrast to the above approaches, we propose a method to produce an exact synchronization between self-excited systems without coupling. By our method, a self-excited system and its copies fall into an exact synchronization in finite time by simply adding noise. The method are motivated by mathematical theories of random dynamical systems studied by Arnold et al. [810]. They showed that parametric noise destroys Hopf bifurcation of the Brusselator [9]. From engineering point of view, we interpret the destruction as a decay of dependency of initial conditions and derive our concept of synchronization.

In this paper, we first demonstrate how added noise destroys Hopf bifurcations and then define a van der Pol-type synchronization system and a nonlinear retarded-type synchronization system. The result shows that both the systems are well synchronized simply by adding noise and that quasi-periodic response of a periodically forced system can also be synchronized in the same way.

\section{Random invariant measures}

2.1 Random dynamical systems Let us consider a nonlinear stochastic differential equation (SDE):

$$
d x=f(x) d t+g(x) d W_{t}, \quad x \in \mathbb{R}^{d}, t \in \mathbb{R}
$$

where $W_{t}$ is a standard Brownian motion. Stochastic counterparts of deterministic fixed points are defined as follows [8].

Let $\Omega$ be the space of continuous functions $\omega: \mathbb{R} \rightarrow$ $\mathbb{R}$ which satisfy $\omega(0)=0$, let $(\Omega, \mathcal{F}, \mathbb{P})$ be the canonical Weiner space, and $\theta_{t} \omega(\cdot) \equiv \omega(t+\cdot)-\omega(t)$. Then, $\left(\Omega, \mathcal{F}, \mathbb{P},\left(\theta_{t}\right)_{t \in \mathbb{R}}\right)$ is an ergodic metric dynamical system which drives the $\operatorname{SDE}(1)$, and $W_{t}(\omega):=\omega(t)$. The solution $x(t, \omega)=\varphi(t, \omega) x_{0}$ has cocycle property $\varphi(t+s, \omega)=\varphi\left(t, \theta_{s} \omega\right) \circ \varphi(s, \omega) s, t \in \mathbb{R}, \omega \in \Omega$ where $\phi(0, \omega)=$ id.

2.2 Invariant measures A random probability measure $\omega \mapsto \mu_{\omega}$ is said to be invariant under $\varphi$ if

$$
\varphi(t, \omega) \mu_{\omega}=\mu_{\theta_{t} \omega}, \quad \forall t \in \mathbb{R} \quad \text { (almost surely) }
$$

The random variable $\mu_{\omega}$ is called a random invariant measure (RIM) and can be regarded as a stochastic fixed point. In contrast to the deterministic fixed point, a sample value of the function $\mu_{\omega}$ of $\omega$ is fluctuated by another dynamics $\theta_{t}$ even if the function $\mu_{\omega}$ is invariant in the sense of eq. (2). Furthermore, there is a one-to-one correspondence called a pullback:

$$
\lim _{t \rightarrow \infty} \varphi\left(t, \theta_{-t} \omega\right) \rho=\mu_{\omega}
$$

where $\rho$ is a stationary probability density function of the process $x$.

Fig.1 shows a sketch of the RIM corresponding to a stable focus. Imagine that rectangles in the left hand side represent transparent phase planes and that the transparent planes are bundled in the right hand side. In the deterministic case above, the focuses compose a fixed point. By contrast, in the random case below, 

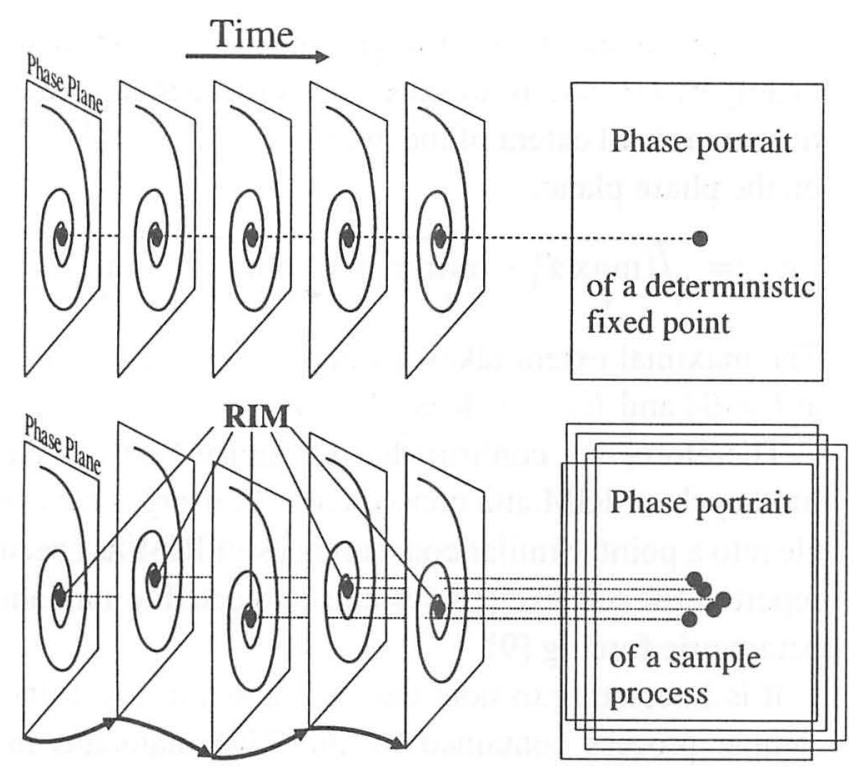

Random Fluctuations

Figure 1: Sketch of a random invariant measure (RIM).

the focuses compose fluctuated points because the RIM $\mu_{\omega}$ is a random variable, i.e. a function of $\omega$ which is fluctuated by another dynamics $\theta_{t}$.

For numerical calculations [11], the probability density function $\rho$ is approximated by

$$
\rho_{n}=\frac{1}{n} \sum_{k=1}^{n} \delta_{X_{k}}
$$

where $X_{k}$ is a numerical solution of the $\operatorname{SDE}(7)$ and $\delta_{(\bullet)}$ is a Dirac measure. Using eqs. (3), (4), the invariant measure is pulled back approximately by

$$
\bar{\mu}_{\omega} \approx \lim _{t \rightarrow \tau} \frac{1}{n} \sum_{k=1}^{n} \delta_{\varphi\left(t, \theta_{-t} \omega\right)} .
$$

Since it is proved that $\bar{\mu}_{\omega} \rightarrow \mu_{\omega}(\tau \rightarrow \infty, n \rightarrow \infty)$, we obtain the approximation $\bar{\mu}$ for sufficiently large $n$ and $\tau$. In practice, we calculate $\mu_{\theta_{t} \omega}=\varphi(t, \omega) \rho$ instead of $\mu_{\omega}=\varphi\left(t, \theta_{-t} \omega\right) \rho$. Because the fixed $\omega$ is not important in our application where temporal changes of the measures are of interest.

To obtain the solution of the SDE (1), we numerically integrate the corresponding random ODE with a random forcing term $\sigma N_{n} / \sqrt{\Delta t}$ by a fourth order Runge-Kutta-Gill method with the step $\Delta t=1 / 64$ where $N_{n}$ is a series of normal random numbers $[0,1]$. The limit $n \rightarrow \infty$ is approximated by the large number $n=80 \times 80=6400$ in what follows.

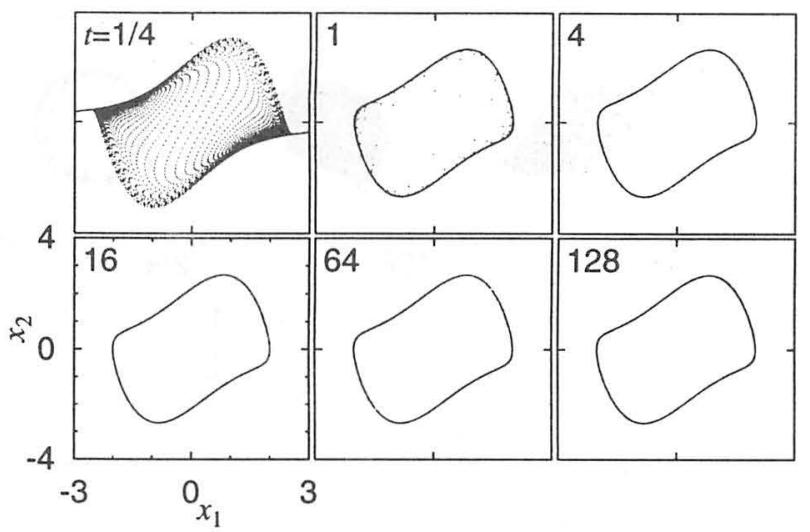

Figure 2: Time history of RIM of the van der Pol system for $(\zeta, \sigma)=(1,0)$ and for $t=1 / 4,1,4,16,64,128$.

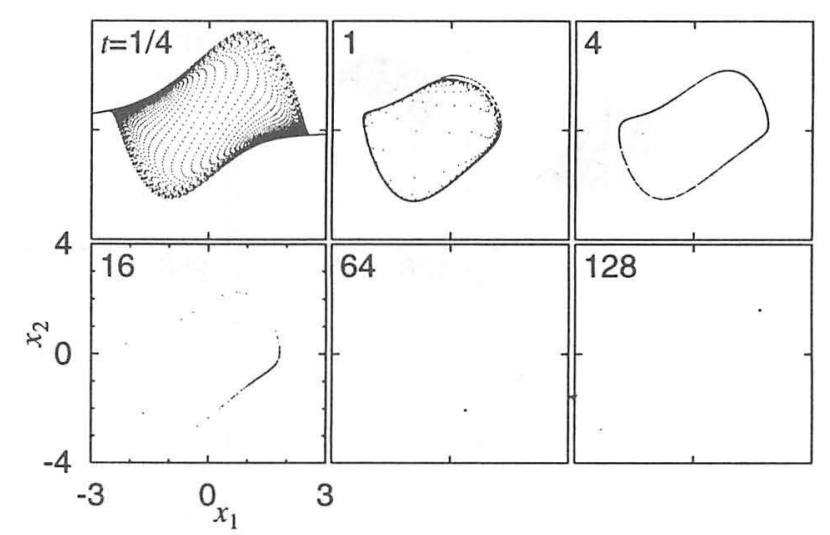

Figure 3: Time history of RIM in the van der Pol system for $(\zeta, \sigma)=(1,0.6)$ and for $t=1 / 4,1,4,16,64,128$.

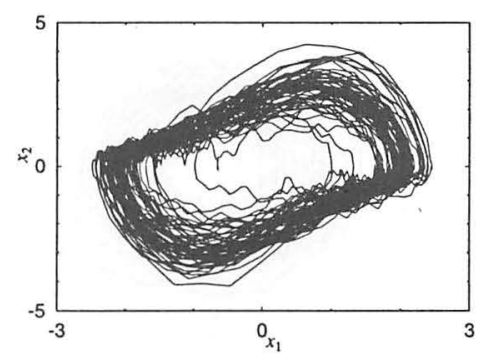

Figure 4: Phase portrait of a sample process of the van der Pol system for $(\zeta, \sigma)=(1,0.6)$.

\section{Destruction of limit cycles by adding noise}

3.1 The van der Pol system Let us consider the van der Pol oscillator subjected to a random excitation:

$$
\begin{aligned}
& d x_{1}=x_{2} d t \\
& d x_{2}=-\zeta\left(x_{1}^{2}-1\right) x_{2}-x_{1}+\sigma d W_{t}
\end{aligned}
$$

where $W_{t}$ is a standard Brownian motion, and $\sigma \in \mathbb{R}$ is an intensity parameter. We assume the fixed parameter $\zeta=1$ while the noise intensity $\sigma$ is assumed to be free.

For noise free case $\sigma=0$, it is well known that the van der Pol system (6) produces a limit cycle due to a Hopf bifurcation. The corresponding RIM is shown 


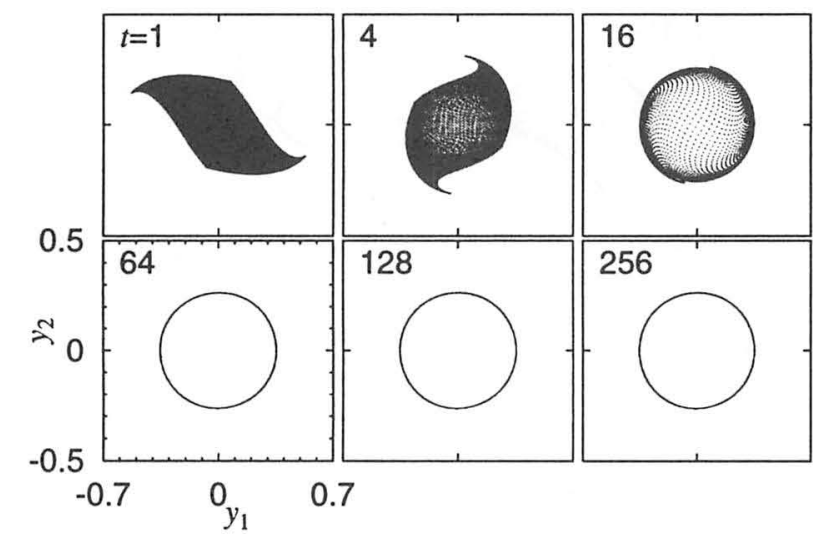

Figure 5: Time history of RIM of the retarded system for $(L, \sigma)=(3 / 16,0)$ and for $t=1,4,16,64,128,256$.

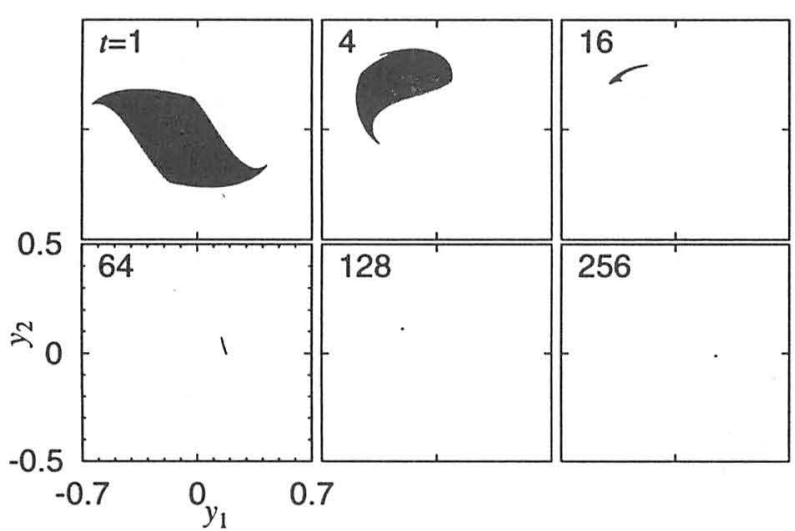

Figure 6: Time history of RIM of the retarded system for $(L, \sigma)=(3 / 16,0.06)$ and for $t=1,4,16,64,128,256$.

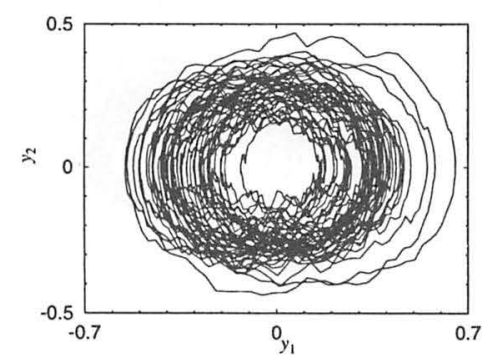

Figure 7: Phase portrait of a sample process of the retarded system for $(L, \sigma)=(3 / 16,0.06)$.

in Fig.2. The RIM is calculated from initial points $\left\{x_{1}^{i}(0), x_{2}^{i}(0)\right\}_{i=1}^{80 \times 80}$ placed at uniform $80 \times 80$ grid points of the rectangle region $[-3,3] \times[-4,4]$ on the $\left(x_{1}, x_{2}\right)$-plane. The solutions $\left\{x_{1}^{i}(t), x_{2}^{i}(t)\right\}_{i=1}^{80 \times 80}$ cycle on a closed curve without intersection with each other. A shape of the closed curve is identical to the corresponding deterministic limit-cycle. In what follows, we refer to the closed curve as a limit cycle of RIM.

Fig. 3 shows a time history of the RIM for noisy case $\sigma=0.6$. The limit cycle of RIM begins to be divided into disconnected segments at $t=4$. The segments are localized and then converged into a point approximately by $t=64$. To evaluate the convergence, we define a maximal extent of the points $\left\{x_{1}^{i}(t), x_{2}^{i}(t)\right\}_{i=1}^{80 \times 80}$ on the phase plane:

$$
E_{x}:=\sqrt{\left(\max _{i} x_{1}^{i}-\min _{i} x_{1}^{i}\right)+\left(\max _{i} x_{2}^{i}-\min _{i} x_{2}^{i}\right)}
$$

The maximal extent takes a value $E_{x}=1.34 \times 10^{-3}$ at $t=64$ and $E_{x}=1.45 \times 10^{-9}$ at $t=64$.

Therefore, we confirm that the noise destroys the limit cycle of RIM and consequently converges the cycle into a point. Similar convergences of RIM has been reported for self-excited systems subjected to random parametric forcing [9].

It is interesting to note that, as shown in Fig.4, the sample process contained by the RIM maintains its cyclic motion even after the RIM is converged into a point as was shown in Fig.3.

3.2 Nonlinear retarded system A quite similar result can also be obtained from a nonlinear retarded system subjected to a random excitation:

$$
\begin{aligned}
& d y_{1}=y_{2} d t \\
& d y_{2}=\left(-\gamma y_{2}-y_{1 L}-\epsilon y_{1 L}^{3}\right) d t+\sigma d W_{t}
\end{aligned}
$$

where $y_{1 L}:=y_{1}(t-L), W_{t}$ is a standard Brownian motion, and $\sigma \in \mathbb{R}$ is an intensity parameter. We assume the fixed parameters $(\gamma, \epsilon, L)=(1,-0.6,3 / 16)$ while the noise intensity $\sigma$ is assumed to be free.

For noise free case $\sigma=0$, the retarded system (7) produces a limit cycle due to a Hopf bifurcation [12]. The corresponding RIM results in the same shape of cycle as the deterministic limit cycle as shown in Fig.5. The RIM is calculated from initial points $\left\{y_{1}^{i}(0), y_{2}^{i}(0)\right\}_{i=1}^{80 \times 80}$ placed at uniform $80 \times 80$ grid points of the rectangle region $[-0.4,0.4] \times[-0.4,0.4]$ on the $\left(y_{1}, y_{2}\right)$-plane.

For noisy case $\sigma=0.06$, the RIM of the retarded system shrinks its shape and then converges to a point approximately by $t=128$ as shown in Fig.6. Fig.7 shows a sample process contained by the RIM. The sample process also maintains its cyclic motion even after the convergence of RIM. The maximal extent $E_{x}$ is obtained as $E_{x}=2.41 \times 10^{-5}$ at $t=128$ and $E_{x}=4.77 \times 10^{-10}$ at $t=256$ for this retarded case.

Therefore, it is obviously found that the noise destroys the Hopf bifurcation of the van der Pol and nonlinear retarded system.

\section{Synchronizing self-excited systems by adding noise}

Let us now look at the result in Fig. 3 and Fig.6 from an engineering point of view. 


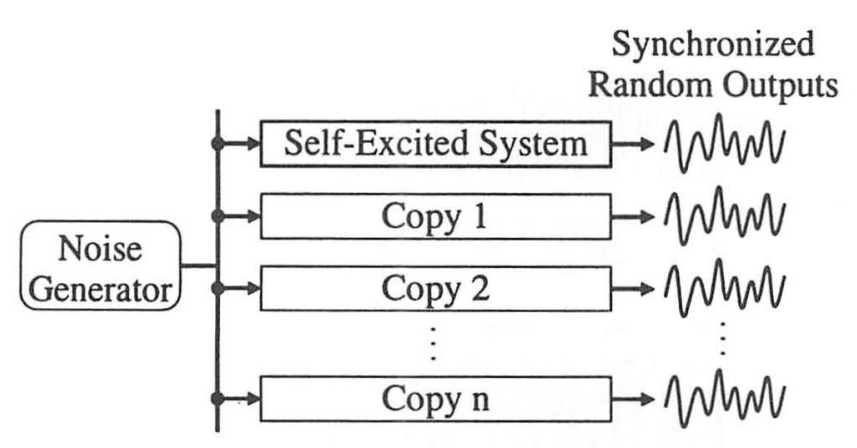

Figure 8: Basic concept of the noise-induced synchronization system. A common noisy input synchronizes a selfexcited system and its copies.

The problem is that a RIM may not be obtainable in experimental situations. For example, $80 \times 80=$ 6400 experiments are required to obtain the result in Fig.6 physically. Moreover, each experiment must be performed with a common sample process of random forcing. This means that the RIM is hardly obtainable from an individual physical oscillator. It follows that the knowledge on the RIM can not be directly applied into an engineering setup for one-time use. Therefore, it might be useful if we could get a knowledge on a sample process which belongs to a RIM which may converge into a random point.

For this purpose, we interpret the convergence of the RIM as a decay of dependency on initial conditions of random responses sharing a common noisy input. Since these random responses are supposed to be elements of the RIM, they must approach a function of time if the RIM is converged into a point.

In view of this, we propose a synchronization system consisting of a self-excited system and its $n$ copies. Our basic concept is schematically shown in Fig.8. In this setup, the $(n+1)$ self-excited systems are synchronized with each other because they are subjected to a common random forcing.

4.1 Van der Pol-type synchronization system To verify our concept, we define a synchronization system consisting of the van der Pol system (6) and its copy:

$$
\begin{aligned}
& u(t)=\sigma w(t), \\
& \dot{x}_{1}=x_{2}, \quad \dot{x}_{2}=-\zeta\left(x_{1}^{2}-1\right) x_{2}-x_{1}+u(t), \\
& \dot{x}_{1}^{\prime}=x_{2}^{\prime}, \quad \dot{x}_{2}^{\prime}=-\zeta\left(x_{1}^{\prime 2}-1\right) x_{2}^{\prime}-x_{1}^{\prime}+u(t)
\end{aligned}
$$

where $w(t)$ is a Gussian noise with zero mean and unit variance.

For noise free case $\sigma=0$, the responses $x_{1}$ and $x_{1}^{\prime}$ are shown in Fig.9(a). The different initial conditions $( \pm 2,0)$ are assigned to the $x_{1}$ and $x_{1}^{\prime}$ system respectively. It is observed that the phase shift between them
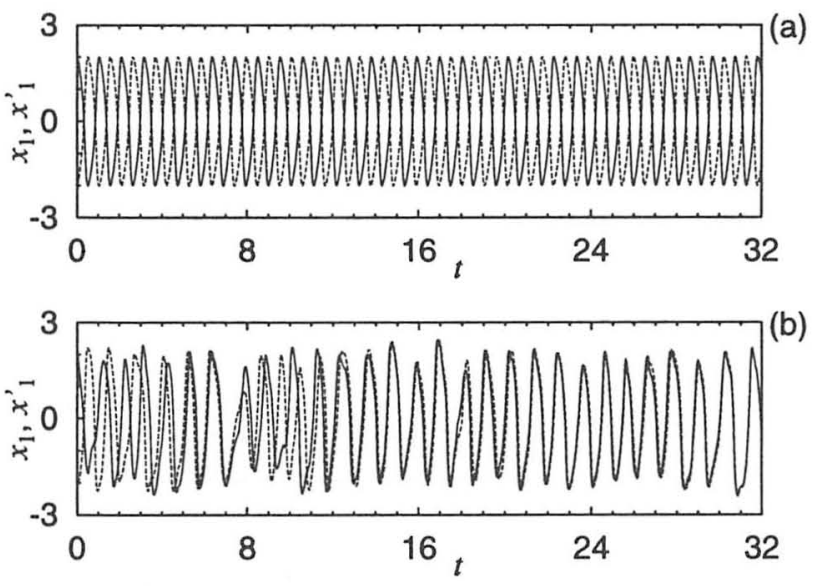

Figure 9: Synchronization in the van der Pol systems for (a) $\sigma=0$; (b) $\sigma=0.6$. The solid and dotted line correspond to the different initial conditions $(-2,0)$ and $(2,0)$ respectively.

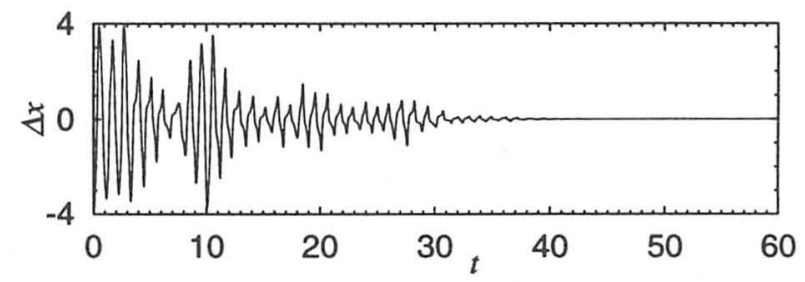

Figure 10: Time series of the difference $\Delta x:=x_{1}^{\prime}-x_{1}$ for $\sigma=0.6$.

becomes constant after some transient state. The result means that the limit cycles has strong and stable dependency on their initial conditions for noise free case. Therefore, the outputs $x_{1}$ and $x_{1}^{\prime}$ are not synchronized with each other for noise free case.

For noisy case $\sigma=0.6$, Fig.9(b) shows the responses $x_{1}$ and $x_{1}^{\prime}$ started from the initial conditions $( \pm 2,0)$ respectively. In contrast to the noise free case, the two responses synchronize with each other. Although the difference between $x_{1}$ and $x_{1}^{\prime}$ exhibits relatively large fluctuations before $t \approx 12$, the difference tends to zero after $t \approx 32$. Fig. 10 shows the difference $\Delta x_{1}:=x_{1}^{\prime}-x_{1}$ as a function of time. $\Delta x_{1}$ converges to zero in a similar way to relaxation oscillations commonly found in the van der Pol-type oscillators.

Therefore, it is clearly found that the proposed system (8) based on our concept in Fig.8 enables us to produce a stable and precise synchronization of the selfexcited system, simply by adding noise.

It is important to note that, the synchronized motions $x_{1}$ and $x_{1}^{\prime}$ are not the original periodic motion any more. They are the random responses of the stochastic self-excited systems(8) as shown in Fig.4.

4.2 Retarded-type synchronization system One can also define a synchronization system consisting of the 

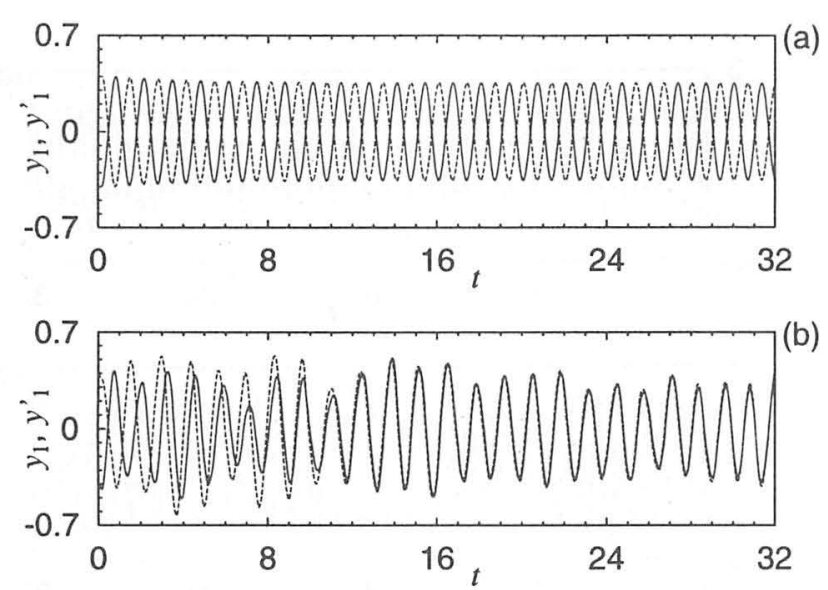

Figure 11: Synchronization in the retarded systems for (a) $\sigma=0$; (b) $\sigma=0.06$. The solid and dotted line correspond to the different initial conditions $(-0.4,0)$ and $(0.4,0)$ respectively.

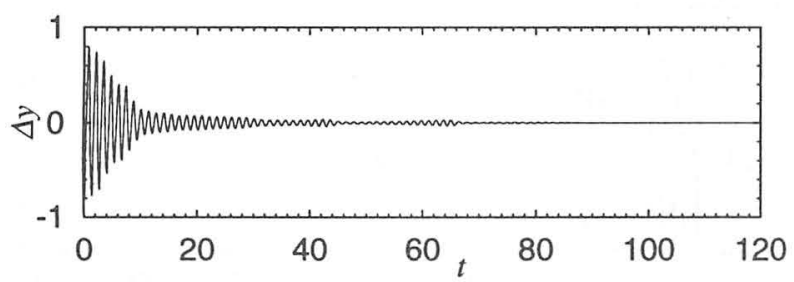

Figure 12: Time series of the difference $\Delta y:=y_{1}^{\prime}-y_{1}$ for $\sigma=0.06$.

retarded system (7) and its copy:

$$
\begin{aligned}
& u(t)=\sigma w(t), \\
& \dot{y}_{1}=y_{2}, \quad \dot{y}_{2}=-\gamma y_{2}-y_{1 L}-\epsilon y_{1 L}^{3}+u(t), \\
& \dot{y}_{1}^{\prime}=y_{2}^{\prime}, \quad \dot{y}_{2}^{\prime}=-\gamma y_{2}^{\prime}-y_{1 L}^{\prime}-\epsilon y_{1 L}^{\prime 3}+u(t) .
\end{aligned}
$$

where $w(t)$ is a Gussian noise with zero mean and unit variance.

For noise free case $\sigma=0$, the responses $y_{1}$ and $y_{1}^{\prime}$ are shown in Fig.11(a). The two responses have the different initial conditions $( \pm 0.4,0)$ respectively. Similar to the van der Pol system, the phase shift between them becomes constant after some transient state. Therefore, the limit cycles has strong and stable dependency on their initial conditions for noise free case.

Fig.11(b) shows the responses $y_{1}$ and $y_{1}^{\prime}$ for noisy case $\sigma=0.06$. The difference $\Delta y_{1}:=y_{1}^{\prime}-y_{1}$ converges to zero as shown in Fig.12. Therefore, The retarded type synchronization system (9) can also produces a stable and precise synchronization, simply by adding noise. This is a quite similar result to that of the van der Pol system.

The above results obviously show that our proposed method to synchronize self-excited systems is effective both for the van der Pol-type implementation (8) and for the retarded-type implementation (9). Our method
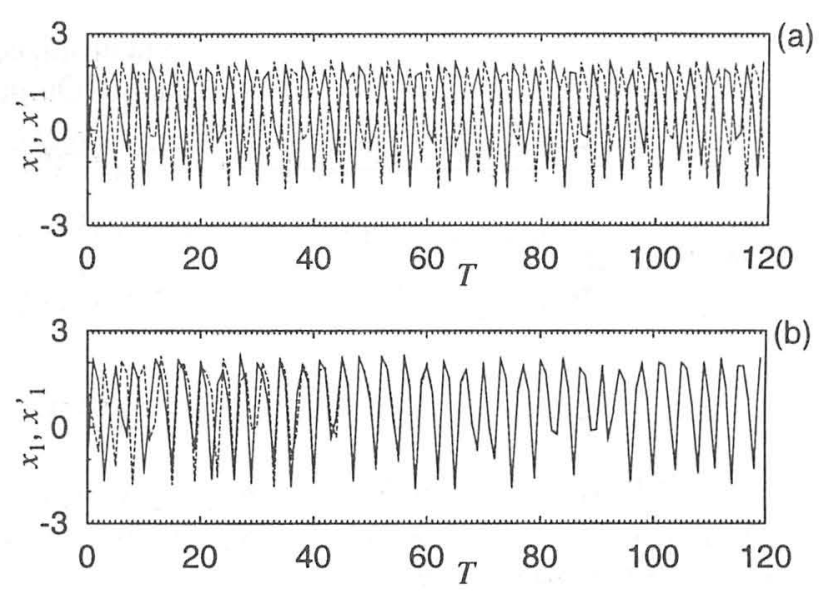

Figure 13: Synchronization in the forced van der Pol systems for (a) $\sigma=0$; (b) $\sigma=0.2$. The solid and dotted line correspond to the different initial conditions $(-1,0)$ and $(1,0)$ respectively.

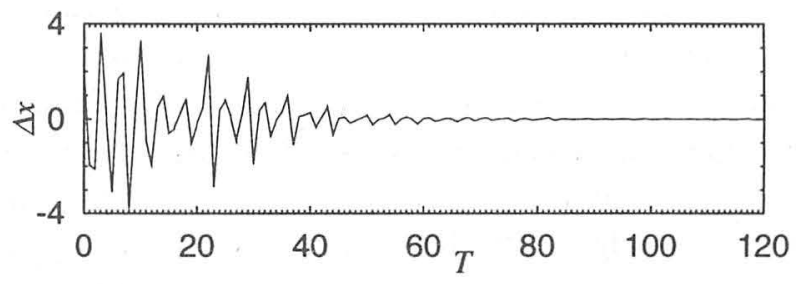

Figure 14: Time series of the difference $\Delta x:=x_{1}^{\prime}-x_{1}$ for $\sigma=0.2$.

is applicable to any number of the copies because there is no interaction between the self-excited systems in our setup proposed in Fig.8.

\section{Synchronizing quasi-periodic systems}

Hopf bifurcations are also generated by another type of dynamics. For example, the Hopf bifurcation of Poincaré maps of periodically forced systems produce a higher dimensional response called quasi-periodic behavior. If our method is effective for responses due to the Hopf bifurcations, the quasi-periodic response of the periodically forced systems is supposed to be synchronized by adding noise. We finally investigate this higher dimensional synchronization by means of a synchronization system consisting of periodically forced van der Pol oscillator and its copy:

$$
\begin{aligned}
& u(t)=\sigma w(t), \\
& \dot{x}_{1}=x_{2}, \\
& \dot{x}_{2}=-\zeta\left(x_{1}^{2}-1\right) x_{2}-x_{1}+A \cos \nu t+u(t), \\
& \dot{x}_{1}^{\prime}=x_{2}^{\prime}, \\
& \dot{x}_{2}^{\prime}=-\zeta\left(x_{1}^{\prime 2}-1\right) x_{2}^{\prime}-x_{1}^{\prime}+A \cos \nu t+u(t)
\end{aligned}
$$

In case of $A \neq 0$, it would seem that rigorous mathematical treatments are not yet available. We intuitively regard $\mu_{\omega}$ as some periodic set in time and introduce a Poincaré map of the $\operatorname{SDE}(10)$ as $\varphi(t, \omega) x \mapsto$ 
$\varphi(t+2 \pi / \nu, \omega) x=\varphi\left(2 \pi / \nu, \theta_{t} \omega\right) \circ \varphi(t, \omega) x$, assuming that $\rho$ is stationary on this mapping in some sense.

Fig.13(a) shows the time series of the Poincaré map of the responses $x_{1}$ and $x_{1}^{\prime}$ for noise free case $\sigma=0$. $T$ is the number of period of the periodic forcing s.t. $t=T \cdot 2 \pi / \nu$. The different initial conditions $( \pm 1,0)$ are given to the two responses respectively. The phase shift between them obviously becomes constant after some transient state. This means that the limit cycle on the mapping also has strong and stable dependency on their initial conditions for noise free case. This is a quite similar result to those of the self-excited systems.

Fig.13(b) shows the result for noisy case $\sigma=0.2$. Fig. 14 shows the difference $\Delta x_{1}:=x_{1}^{\prime}-x_{1}$ as a function of the period $T$. Although convergence of $\Delta x_{1}$ is slower than those of the self-excited synchronization systems, $\Delta x_{1}$ clearly converges to zero.

Therefore, it is confirmed that the quasi-periodictype synchronization system (10) can also produce a stable and precise synchronization, simply by adding noise. The advantage where the method is applicable to any number of the copies also holds in this case.

\section{Conclusion}

We proposed a method that enables us to synchronize the self-excited systems simply by adding noise. For this purpose, we first investigated the destruction of Hopf bifurcations by adding noise. We then interpreted the phenomena as follows:

- The random self-excited systems reach a common response as a function of time if they share a common sample process of random forcing.

Based on this result, we proposed a synchronization system consisting of the self-excited system and its copies sharing a common random forcing and obtained the following results:

- The self-excited system and its copy are synchronized with each other by adding noise

- The synchronization are stably maintained after some transient state which decays in finite time.

- The quasi-periodic system and its copy are also synchronized in the same way.

Our method is applicable to any number of the copies because there is no interaction among the systems in the setup proposed in Fig.8.

The above result leads to the conclusion that well known coupling techniques are not always required to obtain phase synchronization of dynamical systems. Our method will provide another option.

To achive this in practical situations, however, influence of mismatches of the system parameter and noise between the copies on the synchronization and dependency of the noise intensity $\sigma$ on stability of the synchronization should be investigated.

\section{References}

[1] J.T. Ariaratnam and S.H. Strogatz: Phase diagram for the winfee model of coupled nonlinear oscillator, Phys. Rev. Let., 86-19, 4278/4281 (2001).

[2] A. Neiman, L. Schimansky-Geier, A. Cornell-Bell, and F. Moss: Noise-enhanced phase synchronization in excitable media, Phys. Rev. Let., 83-23, 4896/4899 (1999).

[3] C. Zhou, J. Kurths, I.Z. Kiss, and L. Hudson: Noiseenhanced phase synchronization of chaotic oscillator, Phys. Rev. Let., 89-1, 014101/to be appear (2002).

[4] S. Kim, S.H. Park, and H.B. Pyo: Stochastic resonance in coupled oscillator systems with time delay, Phys. Rev. Let., 82-8, 1620/1623 (1999).

[5] D.V.R. Reddy, A. Sen, and G.L. Johnston: Experimental evidence of time-delay-induced death in coupled limit-cycle oscillators, Phys. Rev. Let., 85-16, $3381 / 3384$ (2000).

[6] L. Zhu, A. Raghu, and Y.C. Lai: Experimental observation of superpersistent chaotic transients, Phys. Rev. Let., 86-18, 4017/4020 (2001).

[7] M. Peil, T. Heil, I. Fischer, and W.E. al: Synchronization of chaotic semiconductor laser systems: A vectorial coupling-dependent scenario, Phys. Rev. Let., 88-17, 174101/to be appear (2002).

[8] L. Arnold: Random Dynamical Systems, SpringerVerlag, (1998).

[9] L. Arnold, G. Bleckert, and K.R. Schenk-Hoppe: The stochastic Brusselator: Parametric noise destroys Hopf bifurcation, Stochastic Dynamics, 71/92, Springer-Verlag (1999).

[10] K. Yoshida and K. Sato: Noise-induced chaotic bifurcations of random attractors, Proceedings of the 33rd ISCIE International Symposium on Stochastic Systems Theory and Its Applications, 145/148 (2002).

[11] H. Keller and G. Ochs: Numerical Approximation of Random Attractors, Stochastic Dynamics, 93/115, Springer-Verlag (1999).

[12] K. Sato, S. Yamamoto, T. Okimura, and S. Watanabe: Dynamic motion of a nonlinear mechanical system with time delay (analysis of the self-excited vibration by an averaging method), Trans. JSME Series C (in Japanese), 61-585, 1855/1860 (1995). 